\title{
Charge Fluctuations and Boundary Conditions of Biological Ion Channels: Effect on the Ionic Transition Rate
}

\author{
R. Tindjong*, D. G. Luchinsky*, , P.V.E. McClintock* , I. Kaufman** and \\ R.S. Eisenberg ${ }^{\ddagger}$ \\ ${ }^{*}$ Department of Physics, Lancaster University, Lancaster LA1 4YB, UK. \\ ${ }^{\dagger}$ NASA Ames Research Center, MS 269-3, Moffett Field, CA, 94035, USA. \\ ${ }^{* *}$ VNII for Metrological Service, Gosstandart, Moscow, 119361, Russia. \\ ${ }^{\ddagger}$ Department of Molecular Biophysics and Physiology, Rush Medical college, \\ 1750 West Harrison, Chicago, IL 60612, USA.
}

\begin{abstract}
.
A self-consistent solution is derived for the Poisson-Nernst-Planck (PNP) equation, valid both inside a biological ion channel and in the adjacent bulk fluid. An iterative procedure is used to match the two solutions together at the channel mouth. Charge fluctuations at the mouth are modeled as shot noise flipping the height of the potential barrier at the selectivity site. The resultant estimates of the conductivity of the ion channel are in good agreement with Gramicidin experimental measurements and they reproduce the observed current saturation with increasing concentration.
\end{abstract}

Keywords: ion channels, Poisson-Nernst-Planck equation, Langevin equation, self-consistent approach, charge fluctuation

PACS: $87.16 . \mathrm{Uv}$

\section{INTRODUCTION}

Ion channels are natural nanotubes in cellular membranes which allow the selective passage of ions into and out of the cell, thereby controlling a vast range of biological functions. Much has been learned about their structures in recent years, leading to intensive, fundamental, and applied research in both biology and physics. How ion channels are able to conduct selectively at rates comparable to free diffusion is still not well understood, despite intensive and ever-growing research. In this paper, we discuss the influence of charge fluctuations on an ion's transition through the channel. The model is used for comparison with experimental measurements of the single-channel Gramicidin current at different concentrations. The theory allows for the dependence of the interfacial concentration on the applied potential across the lipid bilayer, and is therefore able to reproduce the ion channel's fast conduction self-consistently. By taking into account the access resistance to the channel, we are able to observe the saturation of the current at higher concentrations. We consider the problem in three steps: (i) definition of the boundary conditions in a self-consistent manner, using coupled solutions of the Poisson and Nernst-Planck (NP) equations in the channel and in the bulk; (ii) theory of the transition rate through the channel in the presence of fluctuations; and finally (iii) comparison with experiment.

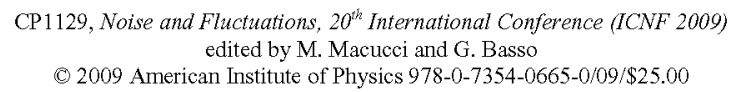




\section{METHODOLOGY}

We use self-consistent Poisson and Nernst-Planck (PNP) equations

$$
\nabla \cdot[\boldsymbol{\varepsilon}(\mathbf{r}) \nabla \tilde{\phi}(\mathbf{r})]=-\left[\sum_{i=1}^{N} z_{j} e \tilde{n}_{j}(\mathbf{r})+e \tilde{p}_{e x}(\mathbf{r})\right],
$$

and combine them with the drift-diffusion NP equation to accommodate the flux of mobile ions

$$
\begin{aligned}
& \frac{\partial \tilde{n}_{j}(\mathbf{r}, t)}{\partial t}=-\nabla \cdot \tilde{J}_{j}=0, \quad(j=1, \ldots, N), \\
& \tilde{J}_{j}=-D_{j}(\mathbf{r})\left[\nabla \tilde{n}_{j}+\frac{z_{j} e}{k_{B} T} \tilde{n}_{j}(\mathbf{r}) \nabla \tilde{\phi}\right] .
\end{aligned}
$$

The potential and the concentrations are thus determined in the bulk (i.e. away from) and close to the mouth of the channel with boundary conditions set at infinity [1]. The PNP equation inside the channel is solved analytically. The solutions obtained in the bulk and in the pore are then combined using an iterative procedure. The current and potential gradient calculated in the pore are utilised to set boundary conditions for the solution in the bulk. The bulk solution is in turn used to set the boundary conditions of the PNP equation in the pore, until convergence is achieved.

We use a 1D approximation of the Poisson equation inside the channel. Applying Brownian dynamics (BD) simulations of ions in the solutions

$$
\begin{aligned}
m_{i} \ddot{\vec{x}_{i}}= & -m_{i} \gamma_{i} \dot{\overrightarrow{x_{i}}}+\left[\frac{q_{i} q_{j}}{4 \pi \varepsilon \varepsilon_{0} r_{i j}^{2}}+\frac{9 U_{0} R_{c}^{9}}{r_{i j}^{10}}\right. \\
& \left.+A U_{0} \exp \left(\frac{R_{i j}-r_{i j}}{a_{e}}\right) \sin \left(2 \pi \frac{R_{i j}-r_{i j}}{a_{w}}-\alpha\right)\right] \frac{\vec{r}_{i j}}{r_{i j}}+\vec{F}_{c h}+\sqrt{2 m_{i} \gamma_{i} k_{B} T} \vec{\xi}_{i}(t),
\end{aligned}
$$

we determine their arrival distribution at the channel mouth. It has been shown that this corresponds to a Poisson distribution [2]. Having estimated the arrival rates of ions at the channel mouth, we use this information to develop a theory of the transition rate through the channel. We assume that the channel is occupied most of the time by only one ion, and that the transition rate of ions through the channel is mainly determined by escape over the potential barrier at the selectivity site. Therefore the effect of the many-body ion dynamics in the bulk on ion motion in the channel is twofold: (i) a delivery of the ions to the channel mouth and (ii) modulation of the channel potential by the charge fluctuations at the channel mouth. Under these physiologically plausible assumptions we separate the ion motion in the channel from the many-body ion dynamics in the bulk. The resultant ion dynamics in the channel may be described by the overdamped Langevin equation,

$$
m \gamma \dot{x}=-\frac{d V(x, t)}{d x}+\sqrt{2 m \gamma k_{B} T} \xi(t),
$$

where the potential $V(x, t)$ has three main contributions: (i) the potential of Coulomb interaction with ions in the bulk solution $V_{C}$; (ii) the electrostatic potential induced by 
interaction with the channel protein $V_{c h}$; (iii) the potential of Coulomb interaction with the wall charge at the selectivity site. By an averaging procedure, the effect of multi-ion motion in the bulk solution is reduced to Coulomb interaction with ions arriving at the channel mouth. The latter process can be viewed as a stochastic Poisson process or as shot noise that modulates the potential barrier for the conducting ion at the selectivity site. We estimate analytically the effect of this potential modulation on the channel conductivity. To quantify the effect of multi-ion motion in the bulk on the conducting ion at the selectivity site, we have simulated the ion's Brownian dynamics in the bulk. The mean arrival time for $\mathrm{Na}^{+}$and $\mathrm{Cl}^{-}$ions at the channel mouth (approximated as a cylindrical section of radius $R$ and length $R$ ) are $\tau^{+}=365 \mathrm{ps}$ and $\tau^{-}=471 \mathrm{ps}$ respectively. These estimates are in agreement with the theoretical estimates in [4]

$$
\tau_{\text {arrival }}=\frac{1}{2 \pi c D R}
$$

where $c$ is the ion concentration and $D$ is the diffusion coefficient. The time evolution of the charge in the channel mouth is a Poisson process with the three main states $+1 e$, 0 , and $-1 e$ Fig. 1(left). As a first approximation we divided the states of the channel potential affected by the charge fluctuations into two effective states: (i) a state of high conductivity, corresponding to $+1 e$, and (ii) a state of low conductivity, corresponding to a charge of either 0 or $-1 e$ at the channel mouth. In this approximation the effect of three states of the potential is taken into account by asymmetry of the transition rates between the two effective states. The corresponding transition rates can be estimated as $\alpha^{ \pm}=1 /\left\langle T_{ \pm}\right\rangle$, where $\left\langle T_{ \pm}\right\rangle$are mean residence time of two effective states, giving $\left(\alpha^{+}\right)^{-1}=0.22 \mathrm{~ns}$ and $\left(\alpha^{-}\right)^{-1}=0.38 \mathrm{~ns}$. The occupation probabilities of these two states are 0.36 and 0.64 respectively.

The effect of charge fluctuations on the channel potential is estimated by solving the Poisson equation for various positions of the conducting ion along the channel axis in the two cases: (i) when there are no other ions at the channel entrances; (ii) when there is one positive ion at the left entrance to the channel.

\section{RESULTS AND CONCLUSION}

Our self-consistent 1D solutions of the PNP equations in the bulk and inside the channel allow us to determine the potential and concentration in both the bulk and the channel. On this basis, we have reconstructed the statistical properties of the charge fluctuations from 3D Brownian dynamics simulations of multi-ion motion in the bulk solution, as shown in Fig. 1(left). We find that the distributions of ion arrival times at the channel mouth are exponential. These charge fluctuations strongly modulate the potential barrier for the conducting ion at the selectivity site due to amplification of electrostatic interactions in long narrow channels of low dielectric constant. These findings have allowed us to model the mean ion transition time through the channel as an ionic escape from the potential well at the selectivity site induced by thermal fluctuations and by modulation of the height of the barrier by stochastic Poisson processes.

We can compare our simulation results with the experimental measurements of the ion current in the Gramicidin channel performed by Andersen et al. [3] in the range 
of concentrations $50 \mathrm{mM}$ to $2 \mathrm{M}$. Fits to the experimental data for two values of the applied potential $75 \mathrm{mV}$ and $100 \mathrm{mV}$ are shown in Fig. 1(right). It can be seen from the figure that the experimental data exhibit a significant saturation of the ion current as a function of concentration, a dependence that is well-reproduced in our simulations. A more accurate model that takes into account the 3 states of the potential is currently under development.
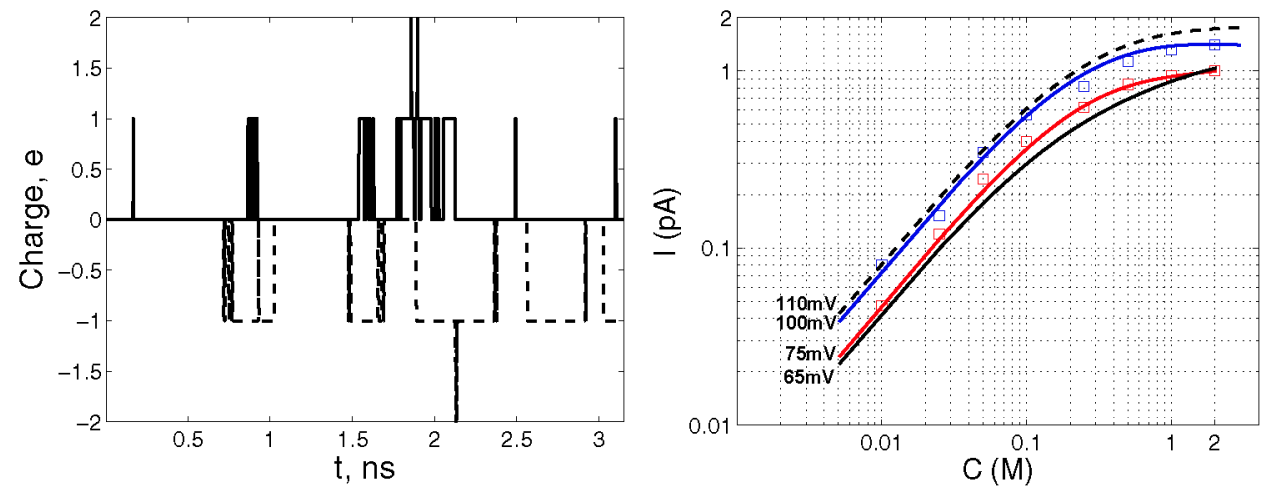

FIGURE 1. Left: Calculated charge fluctuations at the channel mouth. Positive charge is shown by the solid line and negative charge by the dashed one. Right: Comparison of theory with experiment. The current is plotted as a function of $\mathrm{Na}^{+}$ionic concentration for a Gramicidin channel. The data points for the potential difference $75 \mathrm{mV}$ and $100 \mathrm{mV}$ are taken from the single-channel measurements of Andersen et al [3]; the curves are from our theory for a particular set of parameter values given in [1].

\section{ACKNOWLEDGMENTS}

The work was supported by the Engineering and Physical Sciences Research Council (UK), The Russian Foundation for Basic Research, INTAS, and ESF.

\section{REFERENCES}

1. D. G. Luchinsky, R. Tindjong, I. Kaufman, P. V. E. McClintock and R. S. Eisenberg, "Self-consistent solution of the Poisson and Nernst-Planck equations in the bulk and in an ion channel", submitted to Phys. Rev. E.

2. R. Tindjong, D. G. Luchinsky, P. V. E. McClintock, I. Kh. Kaufman and R. S. Eisenberg, "Effect of charge fluctuations on the permeation of ions through biological ion channels", in M.Tacano et al, ed. Noise and Fluctuations, ICNF 2007, AIP vol. 922, Melville, New York, 2007, pp 647-650.

3. O. S. Andersen, R. E. Koeppe, and B. Roux, IEEE Transactions on Nanoscience 4, 10 (2005).

4. Eisenberg R S, Klosek M M and Schuss Z 1995 J. Chem. Phys. 102 1767-1780 
Copyright of AIP Conference Proceedings is the property of American Institute of Physics and its content may not be copied or emailed to multiple sites or posted to a listserv without the copyright holder's express written permission. However, users may print, download, or email articles for individual use. 